\title{
An efficient parallel method to compute lithostatic pressure in thermo-mechanical geodynamic models
}

\author{
Anthony Jourdon ${ }^{1}$ and Dave A. May ${ }^{1}$ \\ ${ }^{1}$ Institute of Geophysics and Planetary Physics, Scripps Institution of Oceanography, UC San Diego, La Jolla, CA
}

Correspondence: Anthony Jourdon (jourdon.anthon@gmail.com)

\begin{abstract}
Modelling the lithostatic pressure in the Earth's interior is a common problem in Earth sciences. In this study we propose to compute the lithostatic pressure from the conservation of momentum of a fluid, reduced to the case of a hydrostatic scenario. This results in a partial differential equation (PDE) which can be solved using the classical numerical methods. To illustrate the usefulness of a PDE based formulation to compute the lithostatic pressure we present two 2D models, one with

5 a deformed mesh and one with a radial gravity vector and a concentric density distribution. Moreover, we also present a 3D rift model which use the lithostatic pressure as a time-dependent boundary condition. This model shows a high degree of non-cylindrical deformation resulting from the stress boundary condition that is accommodated by strike-slip shear zones. We compare the result of this numerical model with a traditional rift model employing free-slip boundary conditions to demonstrate the first order implications of considering "open" boundary conditions in 3D thermo-mechanical rift models.
\end{abstract}

\section{Introduction}

In Earth sciences and geodynamic modelling, computing the lithostatic pressure can be essential. Specifically, numerous thermo-mechanical studies use the lithostatic pressure as a type of stress boundary condition (e.g., Baes et al., 2018; Brune, 2014; Brune et al., 2012, 2014, 2017; Chertova et al., 2012, 2014; Ismail-Zadeh et al., 2013; Popov and Sobolev, 2008; Quinteros et al., 2010; Yamato et al., 2008). Moreover, the lithostatic pressure can also be used as an initial guess for the pressure when solving linear or non-linear systems flow problems with iterative methods.

The common approach to compute the lithostatic pressure $P$ is to define a set of depth columns and integrate the rock density $\rho(\boldsymbol{x})$ over each column to obtain the lithostatic pressure at depth. That is, to compute the pressure $P$ at some point $\boldsymbol{x}^{\prime}$, we evaluate the $1 \mathrm{D}$ integral

$P\left(\boldsymbol{x}^{\prime}\right)=\bar{P}_{0}+\int_{\boldsymbol{x}_{s}^{\prime}}^{\boldsymbol{x}^{\prime}} \rho(\boldsymbol{x})\|\boldsymbol{g}(\boldsymbol{x})\| d x$,

where $\boldsymbol{x}_{s}^{\prime}$ is the projection of $\boldsymbol{x}^{\prime}$ onto the surface of the Earth in the direction opposite to the gravity vector $\boldsymbol{g}$ and $\bar{P}_{0}$ is the reference pressure at the surface $\boldsymbol{x}_{s}^{\prime}$. For the case of a constant density and gravity, this expression reduces to $P\left(\boldsymbol{x}^{\prime}\right)=P_{0}+\rho g D$ where $D$ is the distance (depth) given by $D=\left\|\boldsymbol{x}_{s}^{\prime}-\boldsymbol{x}^{\prime}\right\|$ and $g=\|\boldsymbol{g}\|$. When the density is a function of space and gravity is constant, the 1D integral is decomposed into different segments $D_{i}$ and a suitable quadrature rule is applied over each segment. 
For example using a 1-point Gauss quadrature rule we have

$P\left(\boldsymbol{x}^{\prime}\right)=\bar{P}_{0}+\sum_{i} \int_{D_{i}} \rho(\boldsymbol{x}) g d x \approx \bar{P}_{0}+\sum_{i} \rho_{i} g D_{i}$,

where $\rho_{i}$ is the density at the centroid of the segment $D_{i}$. For the case of a uniform mesh with cell edges aligned with the gravity vector, all the cell edges / vertices are located along straight lines which are parallel to the direction of gravity. Hence Eq. (2) can be simply evaluated by traversing along a column associated with a set of cells (or vertices). In this special case, the sub-division of the integral is naturally defined by mesh cells. If the column sweep is performed from the surface to depth, then only a single pass over each cell in a column is required to compute the pressure at any depth within that column by accumulating values from cells at shallower depths. That is if we traverse from segments $i=0,1,2, \ldots, N$ where the segments are ordered such that $D_{i+1}$ is located at greater depth than $D_{i}$, then we have the following sequence $P_{0}=\bar{P}_{0}+\rho_{0} g D_{0}$, $P_{1}=\bar{P}_{0}+\rho_{0} g D_{0}+\rho_{1} g D_{1}=P_{0}+\rho_{1} g D_{1}, \ldots, P_{N}=\bar{P}_{0}+\sum_{i}^{N} \rho_{i} g D_{i}=P_{N-1}+\rho_{N} g D_{N}$.

Although evaluating Eq. (2) may appear simple, its implementing may be inefficient, or algorithmically complex for general use. Below we outline some common use-cases which render the column-wise integration difficult (or expensive):

1. A mesh with cell edges (2D) or faces (3D) which are not aligned with the gravity vector (Figure 1a);

2. An unstructured mesh (Figure 1b);

3. A density structure (or gravity vector) which is spatially varying;

4. A parallel decomposition of the mesh (Figure 1c);

5. Time-dependence in the density, or mesh coordinates which requires continual re-evaluation of the lithostatic pressure.

To compute $P\left(\boldsymbol{x}^{\prime}\right)$ we first have to define the location $\boldsymbol{x}_{s}^{\prime}$. In general this is non-trivial for use-cases 1 . and 2 . If both the density and gravity are constant, then the only complexity associated with meshes identified in points 1 . and 2. relate to computing $\boldsymbol{x}_{s}^{\prime}$. Due to the fact that the path of the integral (i.e. the "column") does not coincide in general with a set of mesh cells or vertices, the line integral must be performed for each point $\boldsymbol{x}^{\prime}$ in the mesh - the single pass approach used in the gravity aligned mesh is not possible. If the density (or gravity) vary in space throughout the domain, the integral must be approximated via a suitable sub-division in space and, or a quadrature rule. Assuming that the density is a piece-wise constant over each cell, the simplest approximation would be to determine the intersection between the line segment $\left[\boldsymbol{x}^{\prime}, \boldsymbol{x}_{s}^{\prime}\right]$ and each cell, and apply a 1-point quadrature rule over the intersecting segment times. In Figures 1 a) and b) we depict the complexity if this procedure for a non-coordinate aligned and an unstructured mesh. When performing simulations in parallel where the mesh is distributed across multiple MPI ranks, even for the case of a uniform mesh aligned with the gravity vector the column-wise integration approach is somewhat complicated. Individual MPI ranks may compute their local contribution to the sum of accumulated pressures, however the final pressure requires a partial sum to be performed over mesh sub-domains which intersect the 1D line integral. The global reduction (taken MPI ranks overlapping with each 1D line integral) is complicated to define for mesh types identified in points 1 . and 2. Lastly, if the lithostatic pressure is to be used as a boundary condition in a mechanical model, 

the efficiency of the implementation used to compute the lithostatic pressure is important.

For these reasons, we propose an efficient, mesh and numerical method (finite elements, finite differences, finite volumes, etc...) independent way to compute the lithostatic pressure for all scenarios 1-5 above by solving a partial differential equation (PDE) for a hydrostatic equilibrium fluid. We also present thermo-mechanical numerical models and static numerical models applied to Earth sciences and geodynamics to show the usefulness of this approach.

\section{PDE based lithostatic pressure formulation}

For an incompressible fluid in a domain $\Omega$, the conservation of momentum is given by the Navier-Stokes equation:

$\rho\left(\frac{\partial \boldsymbol{v}}{\partial t}+\boldsymbol{v} \cdot \nabla \boldsymbol{v}\right)=\nabla \cdot \boldsymbol{\tau}-\nabla P+\rho \boldsymbol{g}$

where $\boldsymbol{v}$ is the velocity of the fluid, $P$ is the pressure, $\boldsymbol{\tau}$ the deviatoric stress tensor, $\rho:=\rho(\boldsymbol{x}, t)$ is the density and $\boldsymbol{g}:=\boldsymbol{g}(\boldsymbol{x}, t)$ is the gravity vector and $\boldsymbol{x}, t$ denote the space and time respectively. The incompressibility constraint is given as:

$\nabla \cdot \boldsymbol{v}=0$.

In the context of our problems we will decompose the boundary of the domain into two non-overlapping segments: $\partial \Omega_{\text {surf }}$ along which we will regard as the free surface and prescribe that tangential and normal stresses are zero, i.e. $\boldsymbol{\tau}-P \mathbb{I}=\mathbf{0}$ and $\partial \Omega_{\mathrm{i}}$ which denotes the interior parts of the boundary along which we may impose any valid combination of velocity / stress in the normal and tangential directions. Furthermore, $\partial \Omega=\partial \Omega_{\mathrm{i}} \cup \partial \Omega_{\text {surf }}$ and $\partial \Omega_{\mathrm{i}} \cap \partial \Omega_{\text {surf }}=\emptyset$. The outward pointing unit normal vector to $\partial \Omega$ will be denoted via $\hat{\boldsymbol{n}}$.

To define the lithostatic pressure we make the ansatz that $\boldsymbol{v}=\mathbf{0}$, hence Eq. (3) reduces to the usual hydrostatic equilibrium problem:

$\mathbf{0}=-\nabla P+\rho \boldsymbol{g}$.

and Eq. (4) is trivially satisfied in hydrostatic equilibrium. To solve Eq. (5) we require a single equation for $P$. This is achieved by taking the divergence of Eq. (5):

$\nabla \cdot \nabla P=\nabla \cdot(\rho \boldsymbol{g})$

Taking the divergence of the momentum equation is common practice when studying iso-viscous fluids, and or in the derivation of projection based Navier-Stokes flow solvers (Chorin, 1967). The only distinction here is that we have first assumed that $\boldsymbol{v}=\mathbf{0}$ before taking the divergence of the momentum equations. To close the system the following boundary conditions are applied as they represent Earth-like bodies for a static state:

$P=0$ for all $\boldsymbol{x} \in \partial \Omega_{\text {surf }}$ 
Eq. (7) is a Dirichlet constraint and defines that the reference (or datum) pressure should be zero on the surface of our geological body. This is consistent with the observation that the mean pressure on all points on the surface (above sea level) are approximately equal. Secondly we require that

$\nabla P \cdot \hat{\boldsymbol{n}}=\rho \boldsymbol{g} \cdot \hat{\boldsymbol{n}} \quad$ for all $\boldsymbol{x} \in \partial \Omega_{\mathrm{i}}$.

Eq. (8) is required such that the pressure on $\partial \Omega_{\mathrm{i}}$ is consistent with Eq. (5) in the direction normal to the boundary. We will refer to Eqs. (6), (7) \& (8) as the pressure Poisson problem.

To obtain the pressure Poisson problem we asserted that $\boldsymbol{v}=\mathbf{0}$. However, for non hydrostatic cases (i.e. $\boldsymbol{v} \neq \mathbf{0}$ ) which would arise if there existing gradients in density orthogonal to the gravity vector, it is still possible to compute a pressure using Eq. (6). In this case, the pressure from Eq. (6) would represent (in general) a spatially varying reference pressure. To obtain the total pressure (i.e. that due to fluid motion and gravity), the conservation of momentum and mass must be solved without the assumption that $\boldsymbol{v}=\mathbf{0}$.

In addition, whether the fluid is moving or stationary, the lithostatic pressure computed here should not be confused with the static pressure of Bernoulli's equation. From a fluid mechanics point of view, the lithostatic pressure we compute here would represent the potential energy term in Bernoulli's equation for an inviscid fluid.

\subsection{Weak formulation}

Given a test function $q \in H_{1}^{d}(\Omega)$ where $H_{1}^{d}(\Omega):=\left\{v \in H_{1}(\Omega): v=0\right.$ on $\left.\partial \Omega_{\text {surf }}\right\}$ we require that

$$
\int_{\Omega} q \nabla \cdot \nabla P d V=\int_{\Omega} q \nabla \cdot(\rho \boldsymbol{g}) d V .
$$

100 Applying integration by parts to the left and right hand sides yields

$$
\int_{\Omega} \nabla q \cdot \nabla P d V-\int_{\partial \Omega_{\mathrm{i}}} q \boldsymbol{F} \cdot \hat{\boldsymbol{n}} d S=\int_{\Omega} \nabla q \cdot(\rho \boldsymbol{g}) d V-\int_{\partial \Omega_{\mathrm{i}}} q \rho \boldsymbol{g} \cdot \hat{\boldsymbol{n}} d S,
$$

where the flux $\boldsymbol{F}$ is given by

$$
\boldsymbol{F}=\nabla P
$$

Note that the boundary $\partial \Omega_{\text {surf }}$ does not appear in Eq. (10) since the test function $q$ vanishes along the Dirichlet boundary.

Furthermore, from the definition of the boundary condition in Eq. (10) the two surface integrals on the LHS and RHS cancel. Hence $P \in H_{1}(\Omega)$ is a weak solution of the pressure Poisson problem if

$$
\int_{\Omega} \nabla q \cdot \nabla P d V=\int_{\Omega} \nabla q \cdot(\rho \boldsymbol{g}) d V
$$

is satisfied for all $q \in H_{1}^{d}(\Omega)$. We also note that Eq. (12) only requires $\rho \boldsymbol{g} \in L_{2}(\Omega)$, thus the formulation is valid for cases when the density $\rho$ is discontinuous. 
https://doi.org/10.5194/se-2022-7

Preprint. Discussion started: 28 January 2022

(c) Author(s) 2022. CC BY 4.0 License.

(c) (i)

\subsection{Implementation}

The strong (Eq. (6)) and weak (Eq. (12)) formulations of the pressure Poisson problem can be solved using the standard spatial discretisation techniques, e.g. finite differences or finite elements. Moreover, since the equation is of Poisson type, it is readily amenable to be solved using standard iterative multigrid and/or direct solvers. Lastly, because the formulation is expressed in terms of a PDE, it is also straight forward to compute the lithostatic pressure on parallel computing architecture as we can re-use existing discretisation implementations that support domain decomposition.

\section{Numerical examples}

To show the usefulness of solving a PDE to compute the lithostatic pressure we propose several examples with variable mesh geometries and density distribution. Moreover, we also present a thermo-mechanical model using the lithostatic pressure as a boundary condition for the conservation of momentum that we compare with a thermo-mechanical model using a free-slip boundary condition.

\subsection{D Half annulus}

The 2D half annulus model aims showing the efficiency of the method to compute the lithostatic pressure in a body with a radial gravity vector and concentric density repartition (Figure 2a). This model represents a domain extending from

$$
\left\{\begin{array}{l}
\theta \in\left[-\frac{\pi}{2}, \frac{\pi}{2}\right] \mathrm{rad} \\
r \in\left[R_{e}-2700, R_{e}\right] \mathrm{km}
\end{array}\right.
$$

where $\theta$ is the angle and $\mathrm{r}$ the radius in 2D spherical coordinates, $R_{e}$ is the Earth radius (6375 km) and $R_{e}-2700 \mathrm{~km}$ is the mantle-core boundary. Mapped into Cartesian coordinates gives:

$$
\left\{\begin{array}{c}
x=r \sin (\theta), \\
y=r \cos (\theta) .
\end{array}\right.
$$

The gravity vector points to the centre (Cartesian coordinates: 0,0$)$ and the density is distributed as 5 concentric layers with a constant density in each (Figure 2a). The lithostatic pressure varies from 0 to $130 \mathrm{GPa}$ with a concentric distribution following the density distribution and the gravity vector orientation (Figure $2 b$ ).

\subsection{D Sheared rectangle}

The 2D sheared rectangle model aims showing the result of computing the lithostatic pressure in a deformed mesh not aligned with the gravity vector. The domain extends along:

$$
\left\{\begin{array}{c}
x \in[0,130] \mathrm{km} \\
y \in[-100,0] \mathrm{km}
\end{array}\right.
$$


sheared with an angle of approximately $30^{\circ}$ in the $x$ direction. This model presents a uniform and constant density of 3300 $\mathrm{kg} \cdot \mathrm{m}^{-3}$. The computed lithostatic pressure shows a linear increase from the surface to the bottom of the model (Figure 2c). The zero flux boundary conditions on the $x$ normal faces allows simulating an infinite domain in this direction.

\subsection{Thermo-mechanical model}

\subsubsection{Physical model}

140 To simulate the long term evolution of the deformation of the lithosphere we solve the stationary, non-inertial form of the conservation of momentum

$\nabla \cdot \boldsymbol{\tau}-\nabla P=\rho \boldsymbol{g}$

with $\tau$ the deviatoric stress tensor, $P$ the pressure, $\rho$ the density of the fluid and $g$ the gravitational acceleration vector. In addition the continuity equation for an incompressible fluid is solved

$145 \nabla \cdot \boldsymbol{v}=0$,

with $v$ the velocity of the fluid. Moreover, to consider the temperature variations in the domain the time dependant conservation of energy is solved

$\frac{\partial T}{\partial t}+\boldsymbol{v} \cdot \nabla T=\nabla \cdot(\kappa \nabla T)+\frac{H}{\rho C_{p}}$,

with $T$ the temperature, $t$ the time, $\kappa$ the thermal diffusivity, $H$ the heat source and $C_{p}$ the thermal heat capacity.

The numerical solution of Eqs. (16) \& (17) is obtained using a mixed finite element method which independently discretises the velocity and pressure fields. Hence the numerical velocity and pressure obtained are solutions of the weak form of the Stokes problem, given by

$$
\mathcal{A}(\boldsymbol{w}, \boldsymbol{v})+\mathcal{B}(\boldsymbol{w}, p)+\mathcal{B}(\boldsymbol{v}, q)-\int_{\Gamma_{N}} \boldsymbol{w} \cdot \boldsymbol{T}(\boldsymbol{v}, p) d S=-\int_{\Omega} \rho \boldsymbol{w} \cdot \boldsymbol{g} d V
$$

where $\boldsymbol{w} \in H_{1}(\Omega)$ and $q \in L_{2}(\Omega)$ are test functions for the velocity and pressure respectively, $\Gamma_{N}$ denotes the Neumann boundary, $\boldsymbol{T}$ denotes the traction vector given by $\boldsymbol{T}(\boldsymbol{v}, p)=(\boldsymbol{\tau}(\boldsymbol{u})-p \mathbb{I}) \hat{\boldsymbol{n}}$ with $\hat{\boldsymbol{n}}$ being the outward pointing normal vector from the boundary. The bilinear forms for the Stokes problem are given by (Elman et al., 2014):

$$
\begin{gathered}
\mathcal{A}(\boldsymbol{w}, \boldsymbol{v})=\int_{\Omega} 2 \eta \dot{\boldsymbol{\varepsilon}}(\boldsymbol{w}): \dot{\boldsymbol{\varepsilon}}(\boldsymbol{v}) d V, \quad \dot{\boldsymbol{\varepsilon}}(\boldsymbol{u})=\frac{1}{2}\left[\nabla \boldsymbol{u}+(\nabla \boldsymbol{u})^{T}\right], \\
\mathcal{B}(\boldsymbol{v}, q)=-\int_{\Omega} q \nabla \cdot \boldsymbol{v} d V .
\end{gathered}
$$

Both the Stokes and thermal problem were solved using the parallel finite element code pTat in 3D (May et al., 2014, 2015), which employs a mixed $Q_{2}-P_{1}$ discretisation for velocity and pressure and a $Q_{1}$ for the temperature. The discretisation of the energy equation additionally employed SUPG stabilisation (Brooks and Hughes, 1982). 


\subsubsection{Initial conditions and rheology}

To model the strain localization we use nonlinear visco-plastic rheologies expressed in term of viscosity. The ductile parts of the domain are simulated using an Arrhenius flow law for dislocation creep

$\eta_{v}=A^{-\frac{1}{n}}\left(\dot{\varepsilon}^{I I}\right)^{\frac{1}{n-1}} \exp \left(\frac{Q+P V}{n R T}\right)$,

where $A, Q$ and $n$ are material-defined parameters (see Table 1), $R$ is the universal gas constant, $V$ the activation volume and $\dot{\varepsilon}^{I I}$ the strain rate second invariant computed as

$\dot{\varepsilon}^{I I}=\sqrt{\frac{1}{2} \dot{\varepsilon}_{i j}}$.

The brittle parts of the domain are simulated using a Drucker-Prager yield criterion adapted to continuum mechanics, given by

$\eta_{p}=\frac{C \cos (\phi)+P \sin (\phi)}{\dot{\varepsilon}^{I I}}$,

with $C$ the cohesion of the material, $\phi$ the friction angle.

The modelled domain contains 4 initial flat layers representing the upper continental crust, the lower continental crust, the lithosphere mantle and the asthenosphere mantle respectively (Figure 3a). The upper crust extends from the surface of the domain $(y=0 \mathrm{~km})$ to $y=-25 \mathrm{~km}$ and is modelled with a dislocation creep quartz rheology (Ranalli, 1997). The lower crust takes place from $y<-25 \mathrm{~km}$ to $y=-35 \mathrm{~km}$ and is simulated with a dislocation creep anorthite rheology (Rybacki and Dresen, 2000). The lithosphere mantle lays from $y<-35 \mathrm{~km}$ to $y=-120 \mathrm{~km}$ while the asthenosphere mantle lays from $y<-120$ $\mathrm{km}$ to $y=-450 \mathrm{~km}$. They are both modelled using a dislocation creep olivine flow law (Hirth and Kohlstedt, 2003).

The initial density distribution follows the lithologies and is reported in Table 1 . In addition, the density varies with pressure and temperature following the Boussinesq approximation

$\rho(P, T)=\rho_{0}(1-\alpha T+\beta P)$,

with $\rho_{0}$ the initial density, $P$ is the total pressure computed from the conservation of momentum (Eq. (16)) and continuity equation (Eq. (17)), $\alpha$ the thermal expansion and $\beta$ the compressibility.

Moreover, the initial temperature field is computed as a steady-state solution of the heat equation

$\nabla \cdot(\kappa \nabla T)+\frac{H}{\rho C_{p}}=0$,

using a surface temperature of $T=0^{\circ} \mathrm{C}$ at $y=0 \mathrm{~km}$ and $T=1450^{\circ} \mathrm{C}$ at $y=-450 \mathrm{~km}$. Other thermal parameters are reported in Table 1

\subsubsection{Boundary conditions}

180 To show the influence of the lithostatic pressure boundary condition we compare two rift models. In the reference model, an extension velocity of $v_{x}=1 \mathrm{~cm} / \mathrm{yr}$ is applied on the whole faces of normal $x$, whilst on faces of normal $z$ a free-slip boundary 
Table 1. Physical parameters for the thermo-mechanical rift model.

\begin{tabular}{cccccc}
\hline Parameter & Units & Upper crust & Lower crust & Lithosphere mantle & Asthenosphere mantle \\
\hline$A$ & $\mathrm{MPa}^{-\mathrm{n}} \cdot \mathrm{s}^{-1}$ & $6.7 \times 10^{-6}$ & 13.4637 & $2.5 \times 10^{4}$ & $2.5 \times 10^{4}$ \\
$n$ & - & 2.4 & 3.0 & 3.5 & 3.5 \\
$Q$ & $\mathrm{~kJ} \cdot \mathrm{mol}^{-1}$ & 156 & 345 & 532 & 532 \\
$\phi$ & $\circ$ & 30 & 30 & 30 & 30 \\
$C$ & $\mathrm{MPa}$ & 20 & 20 & 20 & 20 \\
$V$ & $\mathrm{~m}^{3} \cdot \mathrm{mol}^{-1}$ & 0 & $38 \times 10^{-6}$ & $8 \times 10^{-6}$ & $8 \times 10^{-6}$ \\
$C_{p}$ & $\mathrm{~m}^{2} \cdot \mathrm{K}^{-1} \cdot \mathrm{s}^{-2}$ & 850 & 850 & 850 & 850 \\
$\kappa$ & $\mathrm{W} \cdot \mathrm{m}^{-1} \cdot \mathrm{K}^{-1}$ & 2.7 & 2.85 & 3.3 & 3.3 \\
$H$ & $\mu \mathrm{W} \cdot \mathrm{m}^{-3}$ & 1.5 & 0.3 & 0 & 0 \\
$\rho_{0}$ & $\mathrm{~kg} \cdot \mathrm{m}^{-3}$ & 2700 & 2850 & 3300 & 3300 \\
$\alpha$ & $\mathrm{K}^{-1}$ & $3 \times 10^{-5}$ & $3 \times 10^{-5}$ & $3 \times 10^{-5}$ & $3 \times 10^{-5}$ \\
$\beta$ & $\mathrm{Pa}^{-1}$ & $10^{-11}$ & $10^{-11}$ & $10^{-11}$ & $10^{-11}$ \\
\hline
\end{tabular}

condition is applied (Figure 3c). To ensure mass conservation we impose an inflow velocity on the bottom face of normal $y$ to balance any outflow which occurs due to the imposed extension. Along the surface of the model we use a free surface (zero normal stress, zero tangential stress) boundary condition.

The second rift model uses more complex boundary conditions (Figure $3 b$ ). On faces of normal $x$, the domain boundary is decomposed into two subdomains $\partial \Omega=\Gamma_{D} \cup \Gamma_{N}$, with $\Gamma_{D}$ the subdomain on which Dirichlet boundary conditions are applied and $\Gamma_{N}$ the subdomain on which Neumann boundary conditions are applied. The Dirichlet boundary conditions are defined as follows:

$$
\left\{\begin{array}{c}
v_{x}=1 \mathrm{~cm} \cdot \mathrm{yr}^{-1} \text { for } y \in[0,-120] \mathrm{km} \\
v_{y}=0 \mathrm{~cm} \cdot \mathrm{yr}^{-1} \text { for } y \in[0,-120] \mathrm{km} \\
v_{z}=0 \mathrm{~cm} \cdot \mathrm{yr}^{-1} \text { for } y \in[0,-120] \mathrm{km}
\end{array}\right.
$$

with the sign of $v_{x}$ being negative on the face $x_{\min }$ and positive on the face $x_{\max }$. The Neumann boundary conditions are defined as follow:

$\boldsymbol{T}=-P_{l} \hat{\boldsymbol{n}}$ for $y \in(-120,-450] \mathrm{km}$,

where $P_{l}$ is the lithostatic pressure computed with Eq. (5) and $\hat{\boldsymbol{n}}$ the normal vector pointing outward the domain. To account for the density evolution through time due to the deformation and material advection, Eq. (5) is solved at every time step and the Neumann boundary condition described by Eq. (25) is evaluated at every time step. On the faces of normal $z$ the Neumann boundary condition described by Eq. (25) is applied on the whole faces. The bottom of the domain is prescribed a no-slip 
condition $(\boldsymbol{v}=\mathbf{0})$ and the surface of the domain is a free surface where the mesh deforms accordingly to the computed velocity field. These Neumann boundary conditions allow material to flow both in and out through the boundary depending only on the Dirichlet boundary conditions and deformation which occurs inside the modelled domain.

\subsubsection{Lithostatic pressure}

In the context of our finite-element forward model, we also solve the pressure Poisson problem using finite elements. As such, to compute $P_{l}$ we employ the weak formulation given by Eq. (12). In our particular implementation, we employ $Q_{1}$ for $P_{l}$, and these $Q_{1}$ elements overlap the $Q_{2}$ elements used to approximate the velocity.

As a demonstration of the computed $P_{l}$ using this approach, in Figures $2 \mathrm{f}$ and $2 \mathrm{~g}$ we show the lithostatic pressure in our rift model at $12.5 \mathrm{Myr}$ after large deformations that led to mantle exhumation, differential thinning of the continental crust and a variable topography has occurred. In this model, $P_{l}$ was evaluated on a mesh consisting of $256 \times 64 \times 128 Q_{1}$ finite elements on 1024 MPI ranks. The discrete pressure Poisson system was solved using geometric multigrid. As a rough estimate, solving for $P_{l}$ required $\sim 0.2 \%$ of the time required to solve the non-linear viscous flow problem. Obviously this value is strongly dependent on both the physical model (linear viscous versus non-linear viscous) and the implementation details, efficiency of how the discrete flow problem is solved. However, when considering even the simplest flow problem imaginable (i.e. linear iso-viscous flow laws), it does remain true that solving the Poisson problem will be far less expensive than solving either the linear or non-linear viscous flow problem.

\subsubsection{Tectonics evolution}

The model using free-slip boundary conditions displays a cylindrical deformation pattern that could be reduced to a two dimensional model. As shown by the shear zones orientation and strain regime, the deformation is only extensional and perpendicular to the extension direction (Figure $4 \mathrm{a}$ to $4 \mathrm{~d}$ and Figure 5). This strain localization is directly due to the free-slip boundary condition stating that any flow perpendicular to the boundary is prohibited. Moreover, the streamlines clearly show a laminar flow which is parallel to the extension direction in the lithosphere and perpendicular below the rift spreading centre (Figure 6a and $6 b)$.

On the contrary, the model using the lithostatic pressure as a boundary conditions displays a non-cylindrical deformation. While extensional shear zones perpendicular to the extension direction develop in the central part of the domain, the edges of the rift experience oblique and strike-slip deformation (Figure $4 \mathrm{e}$ to $4 \mathrm{~h}$ ). As the extension goes on, the extensional deformation localizes along a spreading centre causing an increasing inflow on the boundaries of the domain with the lithostatic pressure boundary condition (Figure 7c). As a result, near these boundaries the velocity field introduces non-cylindrical features which are accommodated by strike-slip faults (Figure $4 \mathrm{~g}$ and $4 \mathrm{~h}$ ). These strike-slip faults delimit a triangular region terminating on a triple junction between two strike-slip faults and a ridge (RFF triple junction). Along these strike-slip faults, the deformation is partitioned between purely vertical strike-slip shear zones and shallow dipping normal shear zones rooting into the strike-slip shear zones (Figure 5). 
In addition, the lithostatic pressure boundary condition applied below the lithosphere on faces perpendicular to the extension direction allow material to freely advect in and out of the domain in a physically consistent manner. Thus, it results that in the asthenosphere, inflow occurs along these boundaries and convection cells are observed to develop below the lithosphere (Figure 6d).

\section{Discussion}

\subsection{Implication on lithosphere deformation}

Using the lithostatic pressure as a boundary condition produces a velocity field perpendicular to the extension direction in the rift axis. This velocity field introduces non-cylindrical deformation accommodated by oblique and strike-slip structures. The results of this study are very similar to previous studies directly applying an inflow perpendicularly to the extension direction (Le Pourhiet et al., 2018; Jourdon et al., 2020). At the tip of the rift, a triangular region delimited by strike-slip faults or very oblique rift develops to accommodate the oblique velocity field. The similarity of these results shows that using open boundary conditions instead of kinematic boundary conditions in 3D may reveal first order implications for the lithosphere strain localization. In the case of geodynamic systems presenting the characteristics of a propagating rift (or ridge) with oblique and strike-slip deformation at its tip, considering the forces applied by the surrounding material weight could be the first order process at the origin of non-cylindrical deformation.

\section{Conclusions}

In this study we presented a method to compute the lithostatic pressure in which we cast the problem in terms of a partial differential equation (PDE). From a practical standpoint, the PDE approach is generic (it's applicable to all spatial discretisation and on any type of computational grid), efficient and amenable parallel computing environments. From the modelling perspective, the PDE approach has specific advantages for example in models with a variable density structure (stationary or time-dependent), and models which employ the lithostatic pressure as a boundary condition of the flow problem (stationary or time-dependent). Re-evaluating the lithostatic pressure in time-dependent problems is not problematic (even if the mesh deforms) since solving the Poisson problem can be performed using optimal preconditioners (e.g. geometric or algebraic multigrid).

Lastly, we showed in the context of 3D models of continental rifting, using the lithostatic pressure as a boundary condition within the flow problem, resulted in non-cylindrical velocity fields which produced strain localization in the lithosphere along large scale strike-slip shear zones and the formation and evolution of triple junctions.

Code availability. The code pTatin 3D used in this study to produce the 3D thermo-mechanical models is an open source free software licensed under GPL3. The supplementary repository contains the version of the code used to produce the models presented in this study. To 
https://doi.org/10.5194/se-2022-7

Preprint. Discussion started: 28 January 2022

(C) Author(s) 2022. CC BY 4.0 License.

(c) (1)

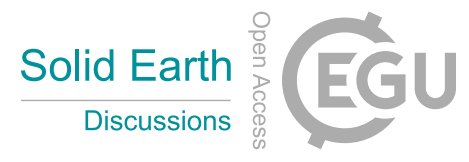

run the same models, user should use the driver named test_driver_checkpoint_fv.app and the options files (.opts) provided in the supplementary files. We also provide FEniCS code to compute the lithostatic pressure in a circular domain and in a square domain with varying densities. The version of EEniCS used is 2016.1.0 and is freely available.

Author contributions. Project conceptualisation: AJ, DAM. Mathematical development: AJ, DAM. Software development: AJ, DAM. Conceived and designed the experiments: AJ, DAM. Performed the experiments: AJ. Analysed the results: AJ. Drafted the manuscript: AJ, DAM. Procured funding, project supervision: DAM.

Competing interests. There are no competing interests

265 Acknowledgements. This project was supported by NSF Award EAR-2121666. The authors gratefully acknowledge the Gauss Centre for Supercomputing e.V. (www.gauss-centre.eu) by providing computing time on the GCS Supercomputer SuperMUC-NG at Leibniz Supercomputing Centre (www.lrz.de) through project pr63qo. 
https://doi.org/10.5194/se-2022-7

Preprint. Discussion started: 28 January 2022

(c) Author(s) 2022. CC BY 4.0 License.

(c) (i)

\section{References}

Baes, M., Sobolev, S. V., and Quinteros, J.: Subduction initiation in mid-ocean induced by mantle suction flow, Geophysical Journal International, 215, 1515-1522, https://doi.org/10.1093/gji/ggy335, 2018.

Brooks, A. N. and Hughes, T. J.: Streamline upwind/Petrov-Galerkin formulations for convection dominated flows with particular emphasis on the incompressible Navier-Stokes equations, Computer methods in applied mechanics and engineering, 32, 199-259, 1982.

Brune, S.: Evolution of stress and fault patterns in oblique rift systems: 3-D numerical lithospheric-scale experiments from rift to breakup, Geochemistry, Geophysics, Geosystems, 15, 3392-3415, https://doi.org/10.1002/2014GC005446.Received, 2014.

Brune, S., Popov, A. A., and Sobolev, S. V.: Modeling suggests that oblique extension facilitates rifting and continental break-up, Journal of Geophysical Research, 117, 1-16, https://doi.org/10.1029/2011JB008860, 2012.

Brune, S., Heine, C., Pérez-Gussinyé, M., and Sobolev, S. V.: Rift migration explains continental margin asymmetry and crustal hyperextension, Nature Communications, 5, 1-9, https://doi.org/10.1038/ncomms5014, 2014.

Brune, S., Heine, C., Clift, P. D., and Pérez-Gussinyé, M.: Rifted margin architecture and crustal rheology: Reviewing Iberia-Newfoundland, Central South Atlantic, and South China Sea, Marine and Petroleum Geology, 79, 257-281, https://doi.org/10.1016/j.marpetgeo.2016.10.018, 2017.

Chertova, M. V., Geenen, T., Van Den Berg, A., and Spakman, W.: Using open sidewalls for modelling self-consistent lithosphere subduction dynamics, Solid Earth, 3, 313-326, https://doi.org/10.5194/se-3-313-2012, 2012.

Chertova, M. V., Spakman, W., Geenen, T., Van Den Berg, A., and van Hinsbergen, D. J. J.: Underpinning tectonic reconstructions of the western Mediterranean region with dynamic slab evolution from 3-D numerical modeling, Journal of Geophysical Research, Solid Earth, 119, 5876-5902, https://doi.org/10.1002/2014JB011150, 2014.

Chorin, A. J.: The numerical solution of the Navier-Stokes equations for an incompressible fluid, Bulletin of the American Mathematical Society, 73, 928-931, https://doi.org/10.1090/S0002-9904-1967-11853-6, 1967.

Elman, H. C., Silvester, D. J., and Wathen, A. J.: Finite elements and fast iterative solvers: with applications in incompressible fluid dynamics, Numerical Mathematics and Scie, 2014.

Hirth, G. and Kohlstedt, D. L.: Rheology of the Upper Mantle and the Mantle Wedge: A View from the Experimentalists, Geophysical Monograph, 138, 83-105, 2003.

Ismail-Zadeh, A., Honda, S., and Tsepelev, I.: Linking mantle upwelling with the lithosphere decent and the Japan Sea evolution: A hypothesis, Scientific Reports, 3, 1-7, https://doi.org/10.1038/srep01137, 2013.

Jourdon, A., Le Pourhiet, L., Mouthereau, F., and May, D.: Modes of Propagation of Continental Breakup and Associated Oblique Rift Structures, Journal of Geophysical Research: Solid Earth, 125, 1-27, https://doi.org/10.1029/2020JB019906, 2020.

Le Pourhiet, L., Chamot-Rooke, N., Delescluse, M., May, D. A., Watremez, L., and Pubellier, M.: Continental break-up of the South China Sea stalled by far-field compression, Nature Geoscience, https://doi.org/10.1038/s41561-018-0178-5, 2018.

May, D. A., Brown, J., and Le Pourhiet, L.: pTatin3D : High-Performance Methods for Long-Term Lithospheric Dynamics, Proceeding SC'14 Proceedings of the International Conference for High Performance Computing, Networking, Storage and Analysis:, pp. 274-284, 2014.

May, D. A., Brown, J., and Le Pourhiet, L.: A scalable , matrix-free multigrid preconditioner for finite element discretizations of heterogeneous Stokes flow, Computer Methods in Applied Mechanics and Engineering, 290, 496-523, https://doi.org/10.1016/j.cma.2015.03.014, 2015 . 
https://doi.org/10.5194/se-2022-7

Preprint. Discussion started: 28 January 2022

(C) Author(s) 2022. CC BY 4.0 License.

(c) (1)

305 Popov, A. A. and Sobolev, S. V.: SLIM3D: A tool for three-dimensional thermomechanical modeling of lithospheric deformation with elasto-visco-plastic rheology, Physics of the Earth and Planetary Interiors, 171, 55-75, https://doi.org/10.1016/j.pepi.2008.03.007, 2008.

Quinteros, J., Sobolev, S. V., and Popov, A. A.: Viscosity in transition zone and lower mantle: Implications for slab penetration, Geophysical Research Letters, 37, n/a-n/a, https://doi.org/10.1029/2010gl043140, 2010.

Ranalli, G.: Rheology of the lithosphere in space and time, Geological Society, London, Special Publications, 121, 19-37, 310 https://doi.org/10.1144/GSL.SP.1997.121.01.02, 1997.

Rybacki, E. and Dresen, G.: Dislocation and diffusion creep of synthetic anorthite aggregates, Journal of Geophysical Research: Solid Earth, 105, 26 017-26 036, https://doi.org/10.1029/2000JB900223, 2000.

Yamato, P., Burov, E., Agard, P., Le Pourhiet, L., and Jolivet, L.: HP-UHP exhumation during slow continental subduction: Self-consistent thermodynamically and thermomechanically coupled model with application to the Western Alps, Earth and Planetary Science Letters, 271, 63-74, https://doi.org/10.1016/j.eps1.2008.03.049, 2008. 
https://doi.org/10.5194/se-2022-7

Preprint. Discussion started: 28 January 2022

(C) Author(s) 2022. CC BY 4.0 License.
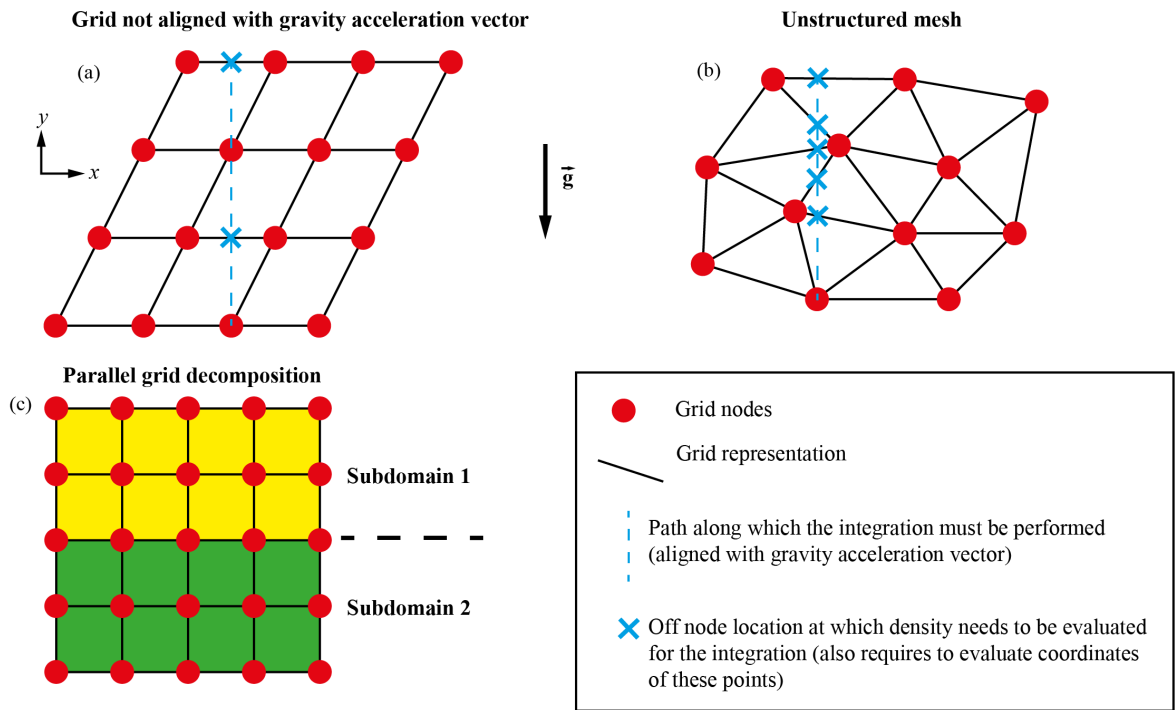

Figure 1. Schematic representation of meshes for which computing an integral in the vertical direction can be challenging. (a) Mesh with a grid not aligned with the the gravity vector. (b) Unstructured mesh. (c) Parallel distribution of a mesh. The dashed blue lines represent the direction along which the integral must be performed. The blue crosses represent the points that have to be evaluated during the integration. 


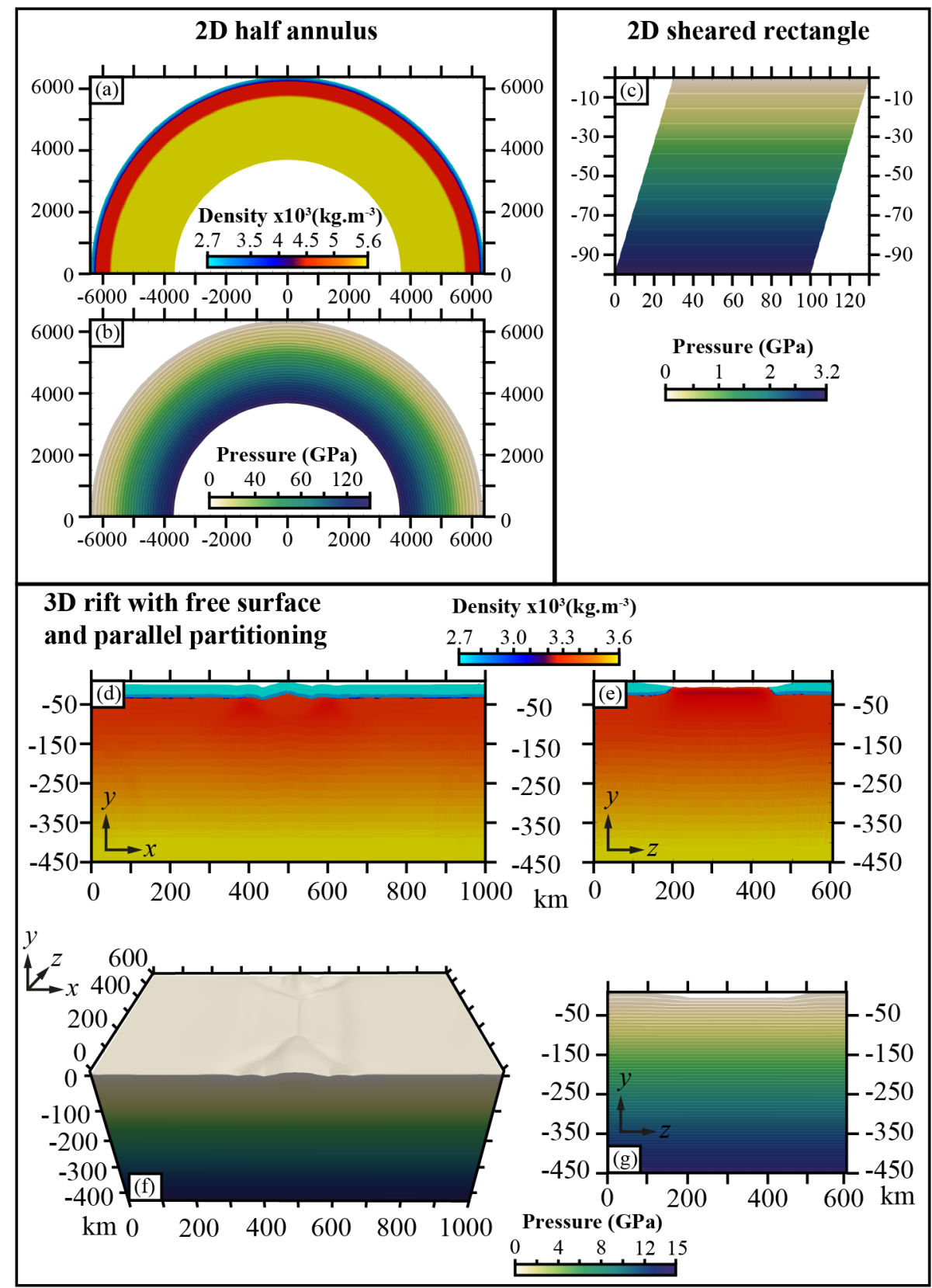

Figure 2. (a) Density structure and (b) lithostatic pressure in a 2D half annulus. The contour lines on the pressure field are plotted every 5 GPa. (c) Lithostatic pressure in a sheared rectangular domain. The contour lines are plotted every $0.25 \mathrm{GPa}$. (d) Density in cross-section view of the 3D rift model with lithostatic pressure boundary condition in the $x-y$ plane at $z=0$. (e) Density in cross-section view in the $z-y$ plane at $x=500 \mathrm{~km}$. (f) $3 \mathrm{D}$ view of the lithostatic pressure. (g) Cross-section view in the $z-y$ plane at $x=500 \mathrm{~km}$ of the lithostatic pressure. The contour lines are plotted every $0.25 \mathrm{GPa}$. 

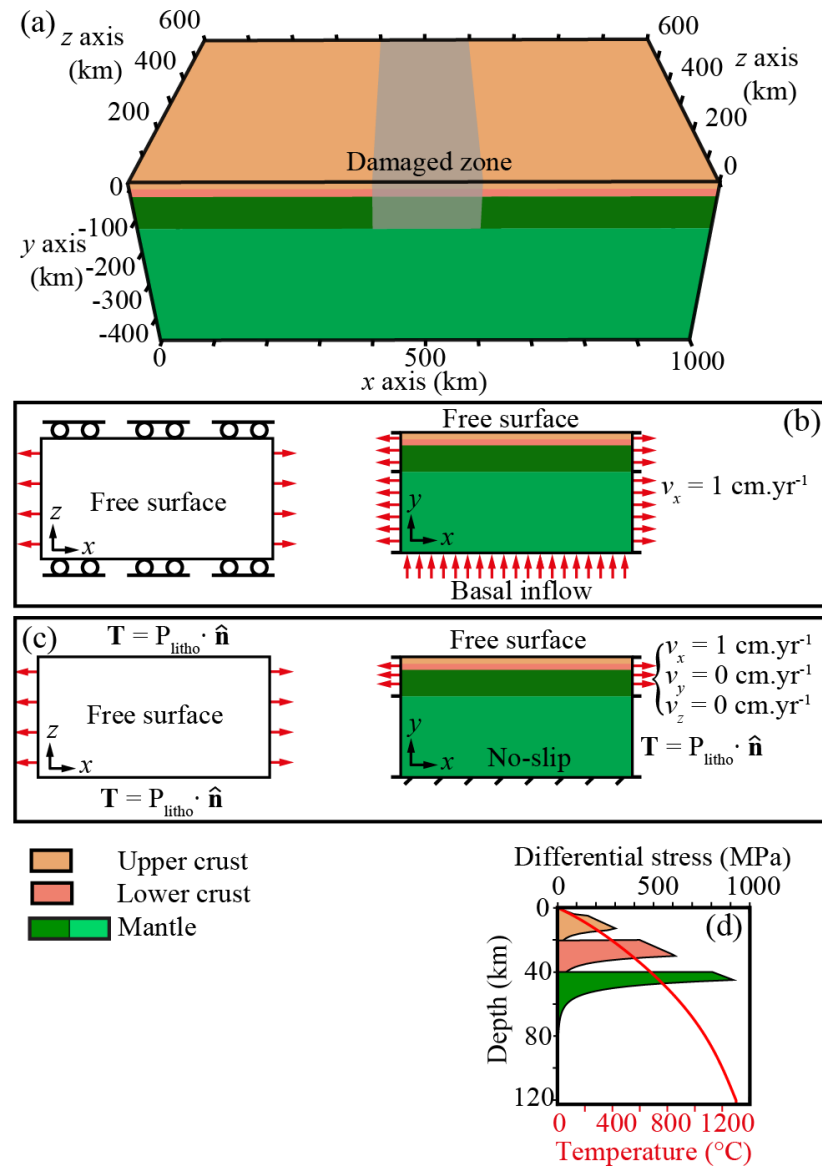

Figure 3. (a) 3D view of the modelled domain. An initial plastic strain with a Gaussian repartition is applied in the central part of the domain in the lithosphere. (b) Map and cross-section view of the boundary conditions for the model with free-slip boundary conditions. (c) Map and cross-section view of the boundary conditions for the model with lithostatic pressure boundary conditions. (d) Yield-stress envelope and initial temperature of the first $120 \mathrm{~km}$. 
Free-slip model
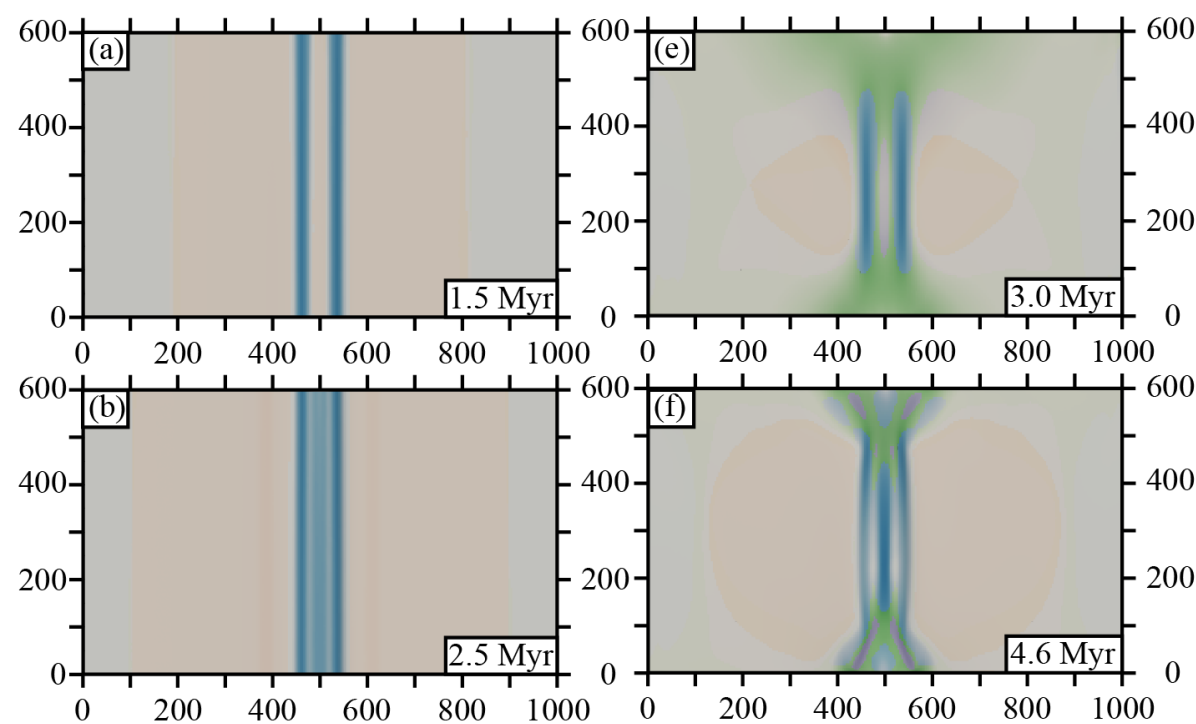

Lithostatic pressure model
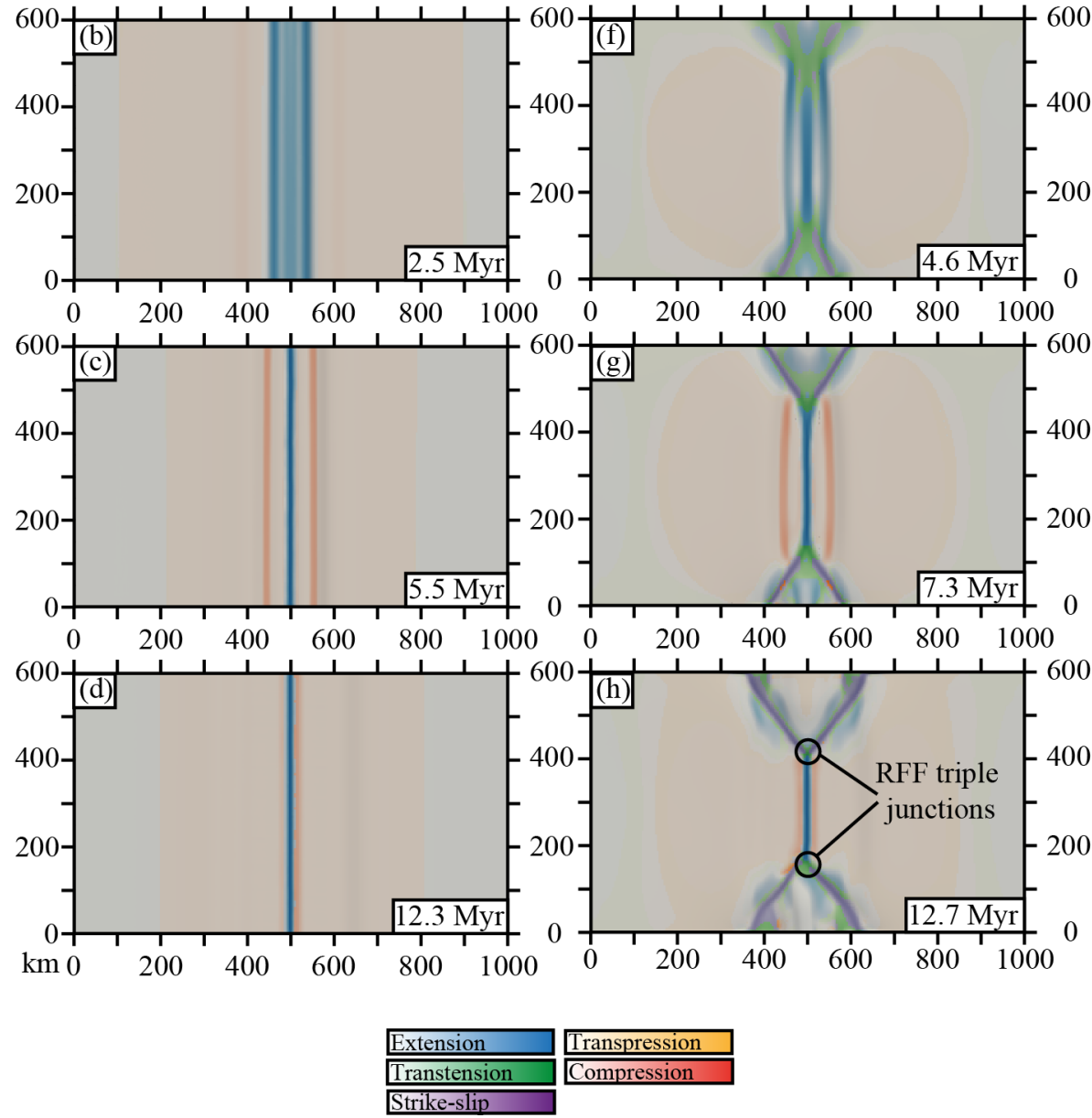

Transpression Compression

Strike-slip

Figure 4. Map view of the strain regime evolution in time and space of the model with (a to d) free-slip boundary conditions and (e to h) lithostatic pressure boundary conditions. 


\section{Free-slip model}
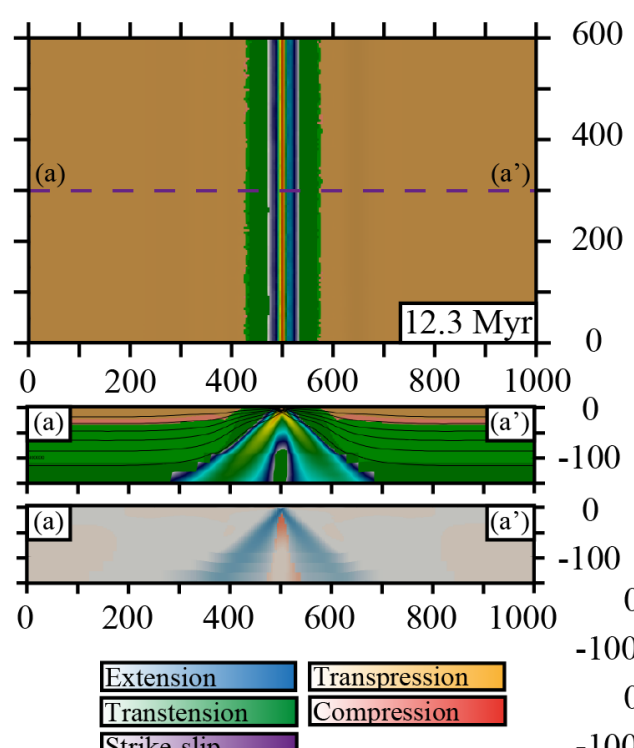

Strike-slip

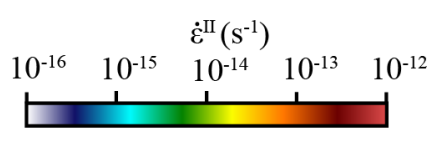

$-100$

0
-100

\section{Lithostatic pressure model}
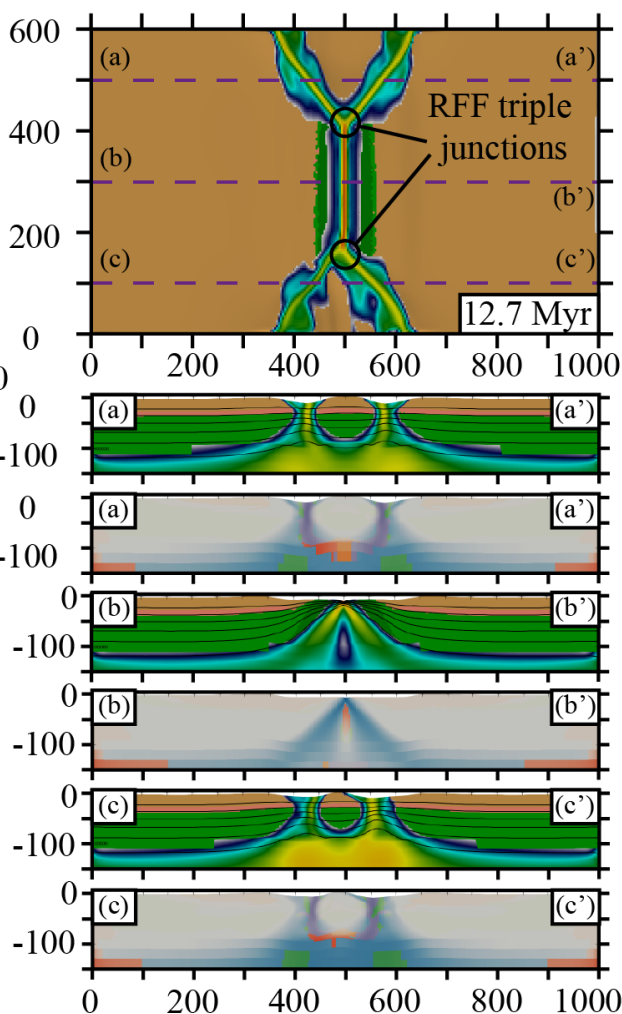

Figure 5. Map view of the models with free-slip (left) and lithostatic pressure (right) boundary conditions. (a)-(a') , (b)-(b') and (c)-(c') lines indicate the cross-sections displayed below. The cross-sections shows the numerical lithologies with strain rate second invariant (Eq. (20)) and the strain regime. 
https://doi.org/10.5194/se-2022-7

Preprint. Discussion started: 28 January 2022

(c) Author(s) 2022. CC BY 4.0 License.

Free-slip BC
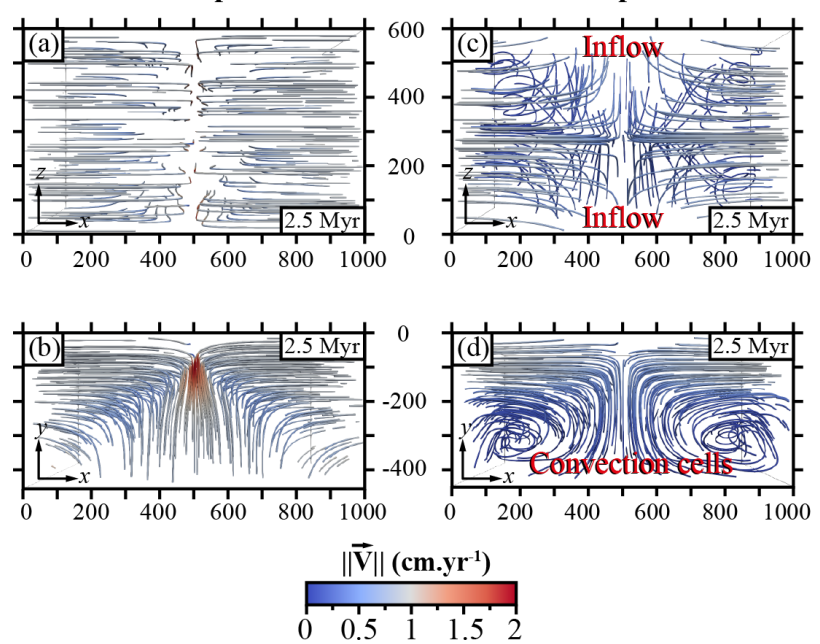

Figure 6. Streamlines at 2.5 Myr of the model with free-slip boundary conditions (left column) and the model with lithostatic pressure boundary conditions (right column). (a) and (c) Downward view of the streamlines. (b) and (d) Side view of the streamlines. The colour scale indicates the norm of the velocity vector. 


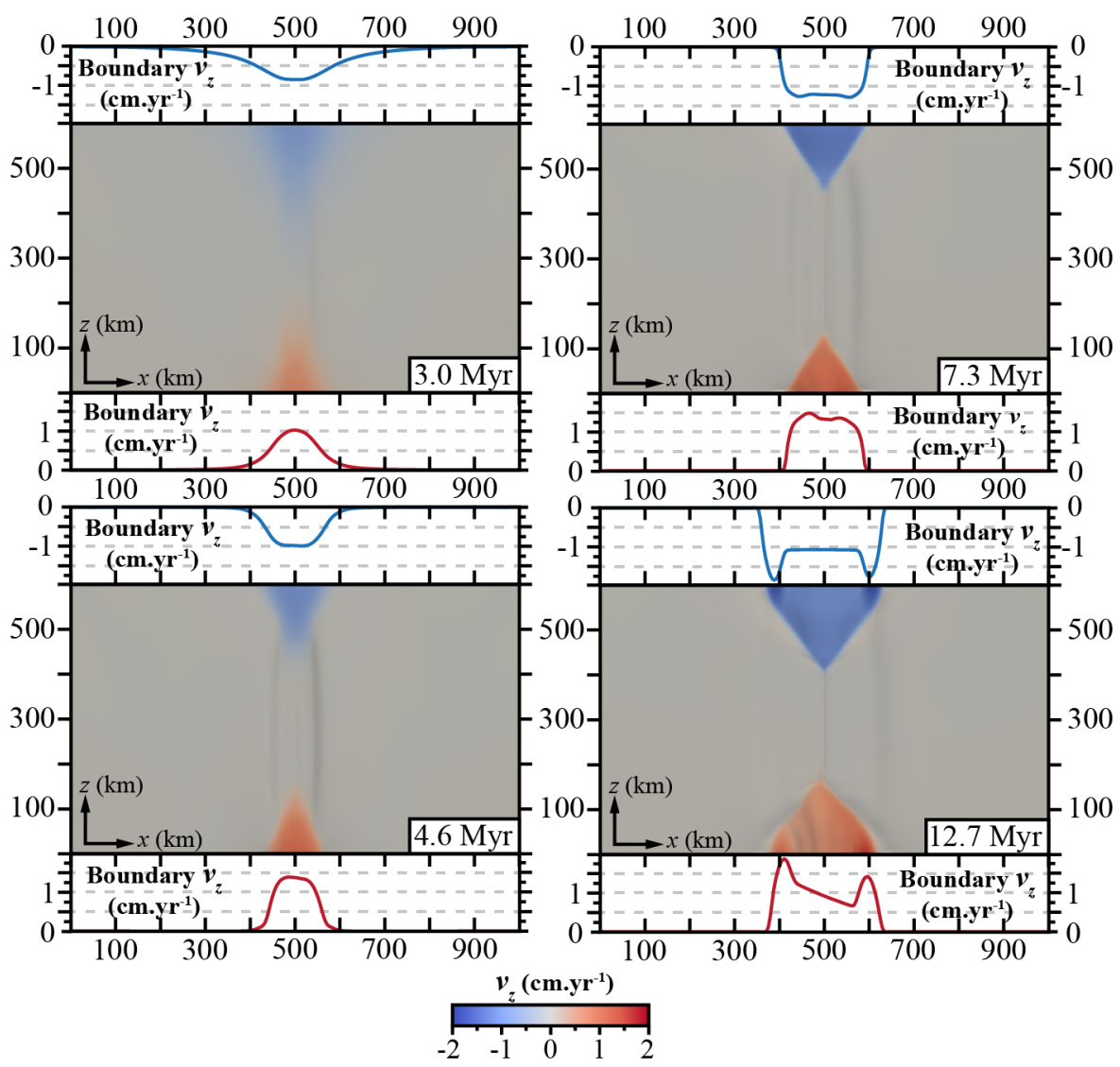

Figure 7. Map view of the $z$ component of the velocity $\left(v_{z}\right)$. Red curves represent the $z$ component of the velocity along the boundary $z_{\text {max }}$ at the surface. Blue curves represent the $z$ component of the velocity along the boundary $z_{\min }$ at the surface. 